\title{
Kapitel VII
}

Jozef Bruk*

\section{Das Präfix be- aus der Sicht der locative alternation: ein Ausgangspunkt für die Raum-Linguistik}

\section{Zusammenfassung}

Im Beitrag wird versucht einige Fragen bezüglich des Präfixes be- zu erörtern. In der deutschen Sprachwissenschaft bemühten sich Günther (1974) oder Eroms (1980) darum, seine Bedeutung und Funktion in Äußerungen zu klären. Unter Verwendung von Talmys figure/ground Hypothese werden einige Grundzüge der bisherigen Forschung dargestellt. Der Schwerpunkt des Beitrags liegt im empirischen Teil, in dem von Paraphrasen für be-Präfixverben, nämlich eines Basisverbs und einer Präposition (z.B. bemalen - malen auf), ausgegangen wird: aufgrund der statistischen Auszählung der Präpositionen wird eine gewisse Raumvorstellung, die für die Semantik des Präfixes be- kennzeichnend sein kann, bestimmt.

Schlüsselwörter: Präfix be-, locative alternation, figure/ground Unterscheidung, Präpositionen, Raumlinguistik.

\begin{abstract}
The article is dealing with so called inseperable German prefix be-. The issue was discussed before by Günther (1974) or Eroms (1980). The aim of the article is to carry out the subsequent research concerning the Eroms work. A next part describes the application of Talmy's hypothesis explaining the figure/ground alignment as the latest approach towards the prefix $b e$ - The main focus of the paper lies in the empirical research based on idea that many be- prefixed verbs have their counterparts created by simplex verbs and prepositions (e. g. bemalen - malen auf).
\end{abstract}

\footnotetext{
* Jozef Bruk, M.A. (Univerzita Mateja Bela v Banskej Bystrici).
} 
A proportion of the prepositions based on statistic report can be useful in defining the space reflected in them.

Keywords: Prefix be-, locative alternation, figure/ground alignment, prepositions, grammars of space.

\section{1. Übersicht über die bisherige Forschung}

\subsection{Interpretation des Präfixes be- als Zusammensetzung}

Die Problematik des Präfixes be- hat eine verhältnismäßig lange sprachwissenschaftliche Tradition hinter sich. Der erste, der sich mit der be- Komposition systematisch befasst hatte, war Hans Christoph Adelung (1774). Als sehr bedeutend werden allerdings auch J. Grimms Ansichten (1826) betrachtet, wobei er be-nicht als Präfix ansieht, sondern von einer Partikel spricht (vgl. Hittmair 1882: 9f.). Im Zusammenhang mit den Verben mit dem Präfix be- Verben wird von J. Grimm (1826, zit. nach Hittmair 1882: 10) Folgendes erwähnt:

Das Verhältnis zwischen be- Kompositum und Objekt müsste, wenn ein nicht zusammengesetztes Verbum gebraucht würde, durch mancherlei Präpositionen,

(1a) Indianer siedeln in der Prärie.

(1b) Indianer besiedeln die Prärie.

(2a) Der Gärtner pflanzt die Rosen auf das Beet.

(2b) Der Gärtner bepflanzt das Beet mit Rosen. ${ }^{1}$

oder mindestens einen andern Kasus bezeichnet werden (Hittmair 1882: 10).

Als weitere Belege für das bereits Erwähnte soll noch Folgendes gelten:

(3a) dienen: der Allgemeinheit, der Bequemlichkeit, dem Fortschritt, der Gerechtigkeit, dem Wohl der Menschheit $\sim$ dienen $+\mathrm{D}$

(3b) bedienen: wir wollen unsere Gäste aufmerksam ; hier wird die Kundschaft gut bedient; kannst du diese Maschine $\sim$ ? bedienen $+\mathrm{A}$

(4a) auftragen: jmdm. etwas ich habe ihm aufgetragen, hier auf dich zu warten; jmdm. eine Arbeit ; das Essen ; jmdm. Grüße (an jmdn.) auftragen + Wem?

(4b) beauftragen: jmdn. ich habe ihn beauftragt, die Bücher abzuholen; jmdn. mit einer Arbeit beauftragen + Wen?

(5a) neiden: jmdm. etwas $\sim$ jmdm. den Erfolg, sein Glück neiden + Wem?

(5b) beneiden: jmdn. ich habe dich glühend beneidet, als dir die Leute zujubelten; ich beneide ihn um seine Energie; beneiden + Wen?

\footnotetext{
${ }^{1}$ Belege nach Eroms (1980: 25f.).
} 
Die angeführten Belege (Wahrig Digital 2007) beweisen eindeutig Grimms Hypothesen. Im Folgenden werden wir uns vorwiegend mit den präpositionalen Fügungen beschäftigen. Die Kasusveränderungen (von Dativ zu Akkusativ) werden erst später bei der Bestimmung der häufigsten Präpositionen erwähnt. Sie sind eher von Randbedeutung für die Untersuchungen, die im vorliegenden Beitrag dargestellt werden.

Eine wichtige Abhandlung zur be-Komposition wurde von Hittmair (1882) verfasst. Hittmair befasst sich nicht nur mit der neuhochdeutschen Entwicklungsperiode, sondern er greift auch zur mittelhochdeutschen Sprachstufe. Seine Monographie ist eher als eine Art Pionierarbeit auf dem Gebiet der Partikel bzw. der Präfixkomposition zu betrachten. Hittmair versucht zum einen das Präfix be- mit den entsprechenden Partikelverben zu paraphrasieren, wie etwa in bewältigen - überwältigen. Zum anderen zählt er die präpositionalen Fügungen aufgrund vermuteter Verwandtschaft von $b e i^{2}$ zu den akkusativfähigen Präpositionen an, auf, über, um, in. Seine Annahme bestätigt er mithilfe des Belegs begehen, der eine mittelhochdeutsche Paraphrasierung an einen gehen zulässt (vgl. Hittmair 1882: 18, 61).

Wie in den obigen Beispielen bereits zu sehen ist, sind die Präpositionen für die Bestimmung der Bedeutung des Präfixes be-von zentraler Bedeutung. Weitere Überlegungen werden mit Hittmairs Zitat, das die Beziehung eines Basisverbs ${ }^{3}$ zu einem be-Verb beschreibt, untermauert:

Die vom Grundwort [Basisverb] des be-Kompositums abhängige und mit einer der genannten Präpositionen gebildete adverbiale Bestimmung wird Objekt des be-Kompositums. Ist das Grundwort [Basisverb] transitiv, so wird außerdem sein Objekt zur instrumentalen Bestimmung des be-Kompositums (Hittmair 1882: 18).

Die Konversion eines ursprünglich intransitiven Satzes wird mittels der Beispielsätze (1a) und (1b) deutlich. Die Konversion eines bereits transitiven Satzes ist in den Beispielsätzen (2a) und (2b) erkennbar und die instrumentale Bedeutung wird mittels der Präposition mit ausgedrückt. Grimms bzw. Hittmairs Beobachtung stellen eine wichtige Annahme für weitere Überlegungen dar. Es muss jedoch erwähnt werden, dass beide Forscher be-Verben stets als Zusammensetzungen von zwei Lexemen betrachteten, deshalb versuchten sie die Funktion und Bedeutung des Präfixes be- ohne enge Beziehung zu dem Basisverb festzulegen. ${ }^{4}$

\footnotetext{
${ }^{2}$ Die Semantik des Präfix be- wird nach Grimm von der vollen Präposition bei abgeleitet.

${ }^{3}$ Die Begriffe Basisverb bzw. Simplexverb werden im Weiteren als gleichwertig betrachtet.

${ }^{4}$ Es ist zu bemerken, dass in Hittmairs Begriffsbestimmung be-Verben als Komposita behandelt werden.
} 


\subsection{Die präfigierten be-Verben: Günthers und Eroms Sichtweise}

Im Gegensatz zur bisherigen Forschungsweise gilt Hartmut Günther (1974) als der erste, der be-Verben als eine Einheit betrachtet. Seine Studie Das System der Verben mit BE- in der deutschen Sprache der Gegenwart kann man als umfangreichen Beitrag zum Gebrauch von be-Verben bezeichnen. Die Gesetzmäßigkeiten, die man bezüglich des Präfixes be- bei Günther finden kann, werden jedoch nicht näher erläutert (vgl. Zifonun 1976: 253; Maylor 2003: 19). Eroms (1980) geht, ähnlich wie Günther, von bestimmten semantischen Gruppen, in die er be-Verben unterteilt, aus. Er nennt, indem er sich auf das Werk von Günther stützt, aufgrund der von ihm gesammelten Datengrundlage 14 Randgruppen. Im Laufe seiner Untersuchung ergänzt er seine Forschung um eine Unterteilung der be-Verben in sechs Hauptgruppen. Die Gruppen I bis $\mathrm{V}$ sind nach dem anwesenden Tiefenkasus geordnet. Zu jeder Gruppe gibt es eine Liste von Verben, die eine Konversion ermöglichen und die zweite, wo keine Konversion möglich ist. Die Gruppen werden wie folgt definiert:

Gruppe Ia: $\mathbf{x}$ be-VERB (volitiv) y mit $\mathrm{z}$ - $\mathbf{x}$ VERB (volitiv) z PRÄPOSITION y (local)

(Der Gärtner bepflanzt das Beet mit Rosen - Der Gärtner pflanzt Rosen auf das Beet)

Gruppe Ib: $\mathbf{x}$ be-VERB (volitiv) y - x VERB (volitiv) PRÄPOSITION y (local)

(Indianer besiedeln die Prärie - Indianer siedeln in der Prärie)

Gruppe II: Ornative be-Verben: $\mathbf{x}$ be-VERB (nomen) $\mathbf{y}-\mathbf{x}$ versieht $\mathbf{y}$ (local) mit einem $\mathrm{z}$ (die Werft bekielt das Schiff - Die Werft versieht das Schiff mit einem Kiel)

Gruppe III: $x$ be-VERB (involitiv/direktional) y (local) - $x$ VERB PRÄPOSITION y

(Das Mädchen beliebeäugelt den Ring - das Mädchen liebeäugelt mit dem Ring)

Gruppe IV: $\mathrm{x}$ be-VERB (emotional) y - x VERB (emotional) (PRÄPOSITION y (local)) (Otto bemäkelt das Essen - Otto mäkelt über das Essen)

Gruppe V: verschiedene, heterogene Untergruppen

Gruppe VI: lexikalisierte be-Verben (vgl. Eroms 1980: 20-25).

Die Begriffe volitiv, involitiv, emotional, ornativ sind sicher noch zu erklären, jedoch stehen sie nicht im Mittelpunkt des raumlinguistisch orientierten Konzepts. ${ }^{5}$ Zudem spricht Maylor (2003: 43) davon, dass ein Affix eine Art

${ }^{5}$ Um diese Begriffe genauer zu klären, müsste man sich an dieser Stelle entweder mit der Tiefenkasus-Theorie näher beschäftigen oder den funktional-typologisch geprägten Ansatz, der beispielsweise von Givón (1984) vorgestellt wird, anwenden. 
der Rückmeldung der Präpositionalphrase zu sein scheint, ohne Rücksicht darauf, ob die Präpositionalphrase ,benefactive', ,instrument' oder ,location' bezeichnet. Eroms (1980: 37) formuliert auf der Basis der Korpusforschung folgende These: „Das Präfix be- [...] ist als stark grammatikalisiertes Morphem aufzufassen, dessen Beschreibung von der Syntax her gerechtfertigt ist."

In Bezug auf die Tatsache, dass be-Verben (wie etwa bemäkeln) die Paraphrasen mittels einer Präposition (mäkeln über) bilden, muss an der Stelle verdeutlicht werden, dass sich der vorliegende Beitrag der syntaktischen Ebene zuwenden wird.

\subsection{Syntaktische Herangehensweise der locative alternation}

Maylor (2003) spricht in seiner Monographie, ${ }^{6}$ die den be-präfigierten Verben gewidmet ist, von der sogenannten locative alternation. Locative alternation verweist auf die Veränderung im Aufbau der Aktanten der

I. transitiven Verben der Bewegung:

(6a) Er schmierte Farbe an die Wand. ${ }^{7}$

(6b) Er beschmierte die Wand mit der Farbe.

II. intransitiven Verben der Bewegung:

(7a) Peter stieg auf den Berg.

(7b) Peter bestieg den Berg.

III. transitiven Verben der Positionierung:

(8a) Peter hängte die Bilder an die Wand.

(8b) Peter behängte die Wand mit den Bildern ${ }^{8}$.

Brinkmann (2007: 3) bezeichnet Verben, die in den Gruppen I, II, III genannt werden, als ,lokative“ Verben, weil sie „eine Beziehung zwischen einer Entität

${ }^{6}$ Der Vorgehensweise liegt die sogenannte lexical template morphology zugrunde.

${ }^{7}$ Die zitierten Belege kommen aus Brinkmann (2007: 1f.).

${ }^{8}$ In Bezug auf Eroms Analyse der be-Verben ist nun zu sagen, dass Eroms jede Hauptgruppe auf die Verben, die einerseits Konversion ermöglichen und anderseits auf die Verben, die keine Konversion (beatmen, begasen, beregnen usw.) erlauben, unterteilt. 
und einem Bezugsraum ${ }^{\prime 9}$ ausdrücken. Die Begriffe Entität und Bezugsraum sind auf eine allgemeinere Dichotomie von ,figure' und ,ground' zurückzuführen. Die Begriffe figure und ground ${ }^{10}$ knüpfen an die gestaltpsychologische Tradition an und wurden von Talmy ([1983] 2000: 314) für sprachwissenschaftliche Zwecke eingeführt. Eigenschaften der genannten Unterscheidung lassen sich wie folgt beschreiben:

figure

bekannte Lokalisierung

kleiner

beweglicher

einfacher

wesentlicher

später im Bewusstsein anwesend

(Talmy 2000: 315f., übers. von J. B.). ground

unbekannte Lokalisierung

größer

fester

zusammengesetzter

im Hintergrund stehender

früher in einer Szene / im Gedächtnis

Wenn man den Beleg (7a) in seiner Paraphrasierung (7b) beachtet, ist noch Folgendes zu berücksichtigen:

Demnach lässt sich der als ground bezeichneten Konstituente die Charakteristik früher und der als figure bezeichneten Konstituente das Adjektiv später zuordnen. Den Ableitungsprozess kann man fortsetzen, und zwar in Richtung logischer Beziehungen oder Kausalitätsbeziehungen. Diese stehen zu den schon abgeleiteten Begriffen früher (vorher) und später (nachher) wie Ursache und Wirkung in dem Sinne, dass die Ursache in der Regel der Wirkung vorausgeht (Tuhárska 2011: 81).

Der obige Beispielsatz und das Zitat lassen uns nun zu Adelungs These (1774) übergehen, die besagt, dass das Präfix be- intransitive Verben zu transitiven macht (vgl. Hittmair 1882: 9). Wenn man alle bisherigen be-Verben näher betrachtet, ist die Akkusativstelle (fast) immer mit einem Objekt besetzt, das konzeptuell ${ }^{11}$ gesehen mit einer Wirkung verbunden ist. Ebenso erklärt Eroms (1980: 23): „Bis auf einige Untergruppen der Gruppen IV, V und VI erfordern die be-Verben im Aktivsatz neben dem Satzsubjekt die obligatorische Besetzung der Akkusativstelle [...]."

${ }^{9}$ Engl.: „Relation between an entity and a spatial region“.

${ }^{10}$ Außer der figure - ground Unterscheidung werden in kognitiv-linguistisch orientierten Ansätzen auch die Begriffe trajector - landmark benutzt. Die gegenseitige Beziehung darf man folgendermaßen darstellen: trajector (<figure) und landmark (< ground). Vgl. dazu auch Langacker (1987), Croft/Cruse (2004).

${ }^{11}$ Der Begriff konzeptuell wird hier in Anlehnung an die figure/ground-Ausrichtung und die Ursache/Wirkung-Unterscheidung benutzt. 


\subsection{Die Grundzüge der Funktion des Präfix be-}

Hinsichtlich der oben angedeuteten Raumbeziehungen wird nun kurz die Vorgehensweise der sogenannten ,Raumlinguistik' bzw. grammars of space diskutiert. Levinson/Wilkins (2006: 15) verwenden im Rahmen von grammars of space den Begriff basic locative construction. Sie erklären ihre Begrifflichkeit aufgrund folgenden Belegs:

(9) Der Apfel ist in der Schale [übers. von J. B.].

Der Beleg aus Levinson/Wilkins greift auf die Analogie mit dem obigen Beispiel (7a): die Nominalphrasen der Apfel und Peter bezeichnen demnach ,figure', wobei die Präpositionalphrasen in der Schale und auf den Berg den ,ground' der Äußerung ausdrücken (vgl. Levinson/Wilkins 2006: 15). Maylor setzt das oben Gesagte in einen unmittelbaren Zusammenhang mit be-Verben, wobei er Talmys Hypothese folgenderweise interpretiert: „Falls die figure/ ground Beziehung vorhanden ist, scheint es eine Regel zu geben, nach der der ,ground' als Bezugspunkt mit einem ,Merkmal der Lokalisierung' betrachtet wird. Ich werde festhalten, dass ,ground' [im Deutschen] typischerweise in einer Präpositionalphrase kodiert ist" ${ }^{\text {"12 }}$ (Maylor 2003: 50, übers. von J. B.).

Croft/Cruse (2004: 56f.) stellen noch fest, dass die figure/ground-Unterscheidung eine asymmetrische Beziehung abbildet. Abschließend zu den figure/ground-Eigenschaften ist zu sagen, dass die erwähnte Beziehung vom Kontext verändert werden kann:

(10) Die Katze [,figure'] ist auf dem Tisch [,ground'].

(11) Ich fand einen Floh [,figure'] auf der Katze [,ground']. ${ }^{13}$

Bezüglich der be-präfigierten Verben bezeichnet Maylor die erwähnte Unterscheidung als Grundvorlage (base template). Die von der Pragmatik ausgeprägten Ansätze zur Syntax (vgl. Schulze 2001: 6) lassen eine gleiche asymmetrische Gewichtung zu, die sich graphisch von der Richtung des Pfeils her ergibt. Dieses Phänomen soll anhand der Belege wie folgt veranschaulicht werden:

(12a) Er lud Heu auf den Wagen.

Er lud Heu $[\rightarrow]$ Wagen

${ }^{12}$ Im Original: „It seems to be a rule of language that, if there is a 'figure'/'ground' relationship between two entities, then the 'ground' is identified by a locative feature. I will maintain that in canonical case the 'ground' is in a locative PP."

${ }^{13}$ Im Original: „The cat ['figure'] is on the table ['ground']. I found a flea ['figure'] on the cat ['ground']." 
(12b) Er belud den Wagen mit Heu.

Er $^{[\rightarrow]}$ lud Wagen *(P) Heu (Maylor 2003: 63).

Demnach lässt sich die oben angeführte Vorgehensweise folgendermaßen darstellen:

(13a) Er baute Häuser (F) auf dem Gelände (G).

(13b) Er bebaute das Gelände (G) mit Häusern (F).

(14a) Er schüttete Sand (F) auf die Straße (G).

(14b) Er beschüttete die Straße (G) mit Sand (F) ${ }^{14}$ (Maylor 2003: 63f.).

Belege wie 13 (a)-(b) und 14 (a)-(b) zeigen deutlich, welche Rolle die Beziehung zwischen figure und ground nach einer Konversion spielt. ${ }^{15}$ Die syntaktische Umstellung bzw. Diathese (vgl. Schulze 2011: 14), die das Präfix beveranlasst, liegt der Verschiebung von Vordergrund zu Hintergrund auf der konzeptuellen Ebene zugrunde. Die besprochene Erscheinung wird sowohl bei Maylor (2003: 64) als auch bei den kognitiv-linguistisch geprägten Quellen (etwa bei Schulze 2001: 31; Langacker 2008: 59) als Foregrounding bezeichnet. ,Foregrounding' tritt insbesondere dann auf, wenn die Unterschiede in den Eigenschaften des Vordergrunds und Hintergrunds geringer sind. Die letztere Annahme bedeutet, dass die Eigenschaften, die normalweise dem Vordergrund zugewiesen werden, auf den Hintergrund übertragen werden können:

(15a) Emil streicht Kitt (V) auf die Stelle (H).

(15b) Emil bestreicht die Stelle (H) mit Kitt (V) (vgl. Eroms 1980: 31).

Die Analyse der Beispielsätze ist in pragmatischer Hinsicht aufschlussreich: Zum einen scheint aus dem funktional geprägten Gesichtspunkt, den sowohl Eroms als auch Maylor übernahmen, dass die Funktion des Präfix be- die Fokussierung bzw. die Topikalisierung des direkten Objekts darstellt. Bezüglich der hinzukommenden Präposition mit behauptet Langacker (2008: 358), dass „Präpositionalphrasen für gewöhnlich zur Kodierung räumlicher Beziehungen (Sie legte es auf das Regal) als auch der Akteure, die außerhalb des Fokus stehen (Sie hat die Suppe mit einer Fliegenklatsche gerührt) [herangezogen werden] ${ }^{\prime 16}$ Da die Fokussierung in dem Satz (15b) auf das Objekt die

${ }^{14}$ Jedoch muss erwähnt werden, dass nach Schulze (2010: 14-19) die oben erwähnte Vorgehensweise in Frage gestellt werden kann.

${ }^{15}$ Die Begriffe figure und ground kann man mit deutschen Gegensätzen mit (szenischen) Vordergrund und Hintergrund ersetzen (vgl. Schulze 2008: 6; Tuhárska 2011: 81).

${ }^{16}$ Im Original: „Prepositional phrases are the usual way of coding both locations (She put it on the shelf) and nonfocused participants, such as instruments (He was stirring the soup with a flyswatter)". 
Stelle erfolgt, bekommt tatsächlich die instrumentale Präpositionalphrase mit Kitt logischerweise weniger Prominenz.

Zum anderen geht der kognitiv-linguistische Ansatz von der Aktivierung des sogenannten CAUSE-Schemas (vgl. Schulze 2007: 8, siehe Abbildung 1) aus. ${ }^{17}$

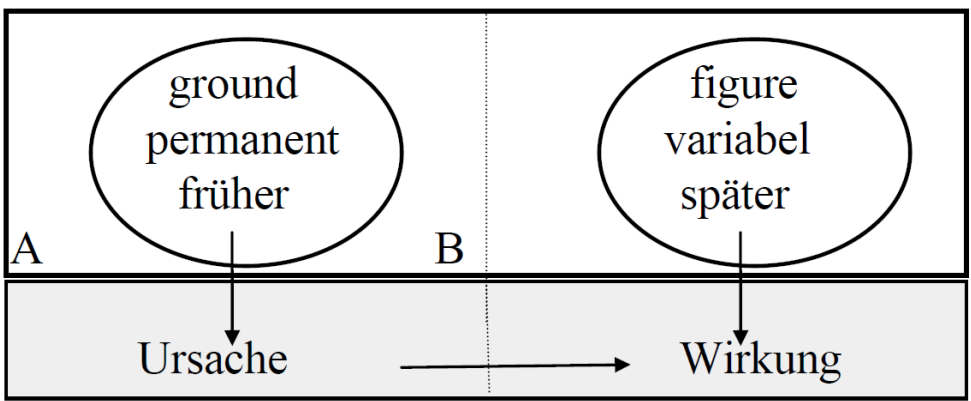

Abbildung 1 Ursache/Wirkung-Verhältnis (Schulze 2000: 49)

Die Abbildung wurde bereits im obigen Zitat von Tuhárska im Zusammenhang mit dem Begriff Kausalität erwähnt: eine kausale Beziehung besteht aus einer Ursache, die eine gewisse Wirkung veranlasst. Wenn man den Beispielsatz Emil bestreicht die Stelle mit Kitt in Betracht zieht, zeigt sich die vorausgehende Stelle (Ursache) deutlich, die erst weiter mit Kitt (Wirkung) bestrichen wird. Schulze (2007: 8) beschreibt das Verhältnis von Vordergrund und Hintergrund zu Ursache/Wirkung (Cause/Effect) folgendermaßen: „Das CAUSE-Schema [C $\rightarrow$ E (Cause $\rightarrow$ Effect)] beruht auf der Metaphorisierung (Funktionserweiterung) des basalen figure/groundSchemas $[\mathrm{F} \rightarrow \mathrm{G}]$. Der Übergang von $\mathrm{F} \rightarrow \mathrm{G}$ zu C $\rightarrow \mathrm{E}$ ist fließend" (ebenda). Darüber hinaus wird mittels der kausalen Beziehung Ursache/Wirkung auch Maylors foregrounding erklärt. Er stellt fest, dass „die natürliche Reihenfolge von den Dingen umgekehrt wird“ (Maylor 2003: 64).$^{18}$ Wenn noch Eroms Position bezüglich der Funktion des Präfixes be- in Betracht gezogen werden soll, muss hier erwähnt werden, dass „die be-Konversion die eine spezifische lokale Relation angebende Präposition [neutralisiert]“ (Eroms 1980: 37).

${ }^{17}$ Vgl. Wildgen (2008: 90). Nach ihm heißt es, dass bei Talmy der Schwerpunkt der Forschung „in der Analyse der Verben der Bewegung, der kausalen Konstruktionen und der verschiedenen Vorstellungssysteme" liegt.

${ }^{18}$ Im Original: „[...] the natural order of things is reversed.“ 


\section{Die Zugangsweise von grammars of space bzw. der Raum-Linguistik in der Frage nach der Bedeutung des Präfix be-}

\subsection{Eine Einführung zum Ausdruck von Positionierung (location $^{19}$ ) hinsichtlich des Präfix be-}

Sowohl bei der Frage nach der Funktion als auch der Frage nach der Bedeutung des Präfix be- ist von den Gesetzmäßigkeiten der locative alternation auszugehen. Das heißt, dass besondere Aufmerksamkeit auf die präpositionalen Fügungen gelenkt wird, die den be-Paraphrasen zugrunde liegen. Bezüglich der räumlichen Beziehungen, ${ }^{20}$ die vorzugsweise die Wortklasse der Präpositionen betreffen und in der statistischen Auszählung des vermuteten Raumes des Präfix be- als prototypisch anzusehen sind, ist Folgendes zu beachten: „Eine Lokalisierung kann entweder als ein präpositionales oder als ein direktes Objekt ausgedrückt werden (Brinkmann 2007: 3, übers. von J. B. $)^{21}$ und dazu noch weiter:

Unsere Kognition benutzt zwar eine hoch-präzise Euklidische Geometrie, aber die Sprache weicht von dieser Geometrie auf bemerkenswerte Art und Weise ab. Diese Abstraktion der Sprache gibt uns Auskunft über die Sprache - nicht aber über die kognitiven Strukturen, die für die Sprachproduktion verantwortlich sind (Levinson 2003: 63, übers. von J. B.). ${ }^{22}$

Es soll die These aufgestellt werden, dass eben das Präfix be- eine bemerkenswerte Art und Weise der Abstraktion abbilden dürfte, da die mittels $b e$-gebildeten Paraphrasierungen auf gewisse lokale Bestimmung zurückzuführen sind. Diesbezüglich hat bereits Hittmair Folgendes festgestellt: „Bei der Beschränktheit des bei auf die sinnliche Bedeutung (nebenbei, beiseite, herbei, hinzu; Grimm bemerkt zur Substantiv-Komposition mit

${ }^{19}$ Vater (vgl. 1996: 41) unterscheidet zwischen statischer Referenz oder Positionierung (Beschreibung der Position eines Objekts) und dynamischer Raumreferenz oder Direktionalisierung (Beschreibung der Verlagerung eines Objekts).

${ }^{20}$ Der deutsche Begriff räumliche Beziehungen ist mit dem englischen spatial relations gleichzusetzen.

${ }^{21}$ Im Original: „The location can be expressed either as a prepositonal object or as the diret object of the verb."

${ }^{22}$ Im Original: „ [...] the sensory and motor systems] of human cognition require highly precise Euclidean metric system of coordinates, and it is clear that language abstracts from these in interesting ways. But this abstraction tell us about language, not the underlying cognitive systems." 
$b \hat{i}-$ und $b i^{-}$, dass $b \hat{\imath}-$ nicht für die Abstraktbildungen angewendet werde) vollzog sich die Differenzierung von be- rasch und gründlich“ (Hittmair 1882: 16).

\subsection{Topologische Positionierung und Annahme eines Referenzrahmens ${ }^{23}$}

Die Raumkonzepte, die englische Präpositionen wie in (in), at (an), on (auf), near (in der Nähe von), between (zwischen) usw. bezeichnen, beziehen sich in der deutschsprachigen Fachliteratur auf die Benennung topologische Positionierung (Vater 1996: 64) und im anglo-amerikanischen Sprachraum auf topology (Levinson 2003: 67). Generell beschreiben die Präpositionen Vorstellungen „wie Identität, echte Inklusion, Differenz, Durchschnitt und Fusion“ (Vater 1996: 66). Levinson (2003: 73) verzeichnet zudem die Vorstellungen wie ,contact' (Berührung), ,vertical relation' (aufrechte Beziehung), ,adhesion' (Anhaften) und ,containment' (Einschluss). Diese begriffliche Inkongruenz wird aufgrund der empirischen Untersuchung eines Raumkonzepts, das durch das Präfix be- beschrieben wird, nachfolgend erörtert.

Eine andere Strategie der Erfassung des Raumes in den Sprachen lässt den Begriff Referenzrahmen bzw. Koordinatensystem einführen. Der Referenzrahmen oder das Koordinatensystem teilt man in zwei Ebenen, nämlich in die aufrechte (,vertical') und die waagerechte (,horizontal') Dimension. In Anlehnung an Schweizer bemerkt Vater (1985: 2) zur Vertikalität und Horizontalität Folgendes:

Für die menschliche Wahrnehmung spielen die vertikale und horizontale Auszeichnung des Raums eine gleichermaßen bedeutende Rolle. Der Fall von Gegenständen, der aufrechte Gang des Menschen, die Himmel-Erde-Relation machen Vertikalität erfahrbar. Der Blickwinkel der Augen vermag in der Horizontalen eine größere Reichweite als in der Vertikalen zu erfassen; das Gehör nimmt Stereo-Effekte, Echo, Schall etc. zur Orientierung und Erfassung räumlicher Konstellationen primär in rechts/links-, vorn/hintenund nah/fern-Differenzierung wahr, und selbst Tastsinn und Eigenbewegung sind primär horizontal ausgerichtet (Vater 1996: 33).

Von hoher Bedeutung ist die Tatsache, dass das Koordinatensystem üblicherweise eine gewisse Winkelangabe ${ }^{24}$ zur Beschreibung der Räumlichkeit eines Gegenstandes einbezieht (vgl. Levinson 2003: 65f.).

${ }^{23}$ Die theoretische Ausgangsposition, die hier beschrieben wird, soll der empirischen Forschung zugrunde liegen.

${ }^{24}$ Der Begriff Winkelangaben wird als mathematische Größe verstanden. In der raumlinguistischen Begrifflichkeit soll hinzugefügt werden, dass diese Winkelangaben zu einer Beschreibung der gerichteten Nachbarschaften, die abhängig von der Position des Objekts sind, 
Levinson macht weiter darauf aufmerksam, dass die topologischen Präpositionen einen umfassenden Bezug auf den Referenzrahmen nehmen können. Wie oben erwähnt wurde, gibt es grundsätzlich Präpositionen, in denen sich keine Winkelangaben widerspiegeln, während sich für eine andere Gruppe ihre Semantik mittels intrinsischer Eigenschaften oder Orientierungsachsen eines Bezugsobjekts ${ }^{25}$ (engl. landmark) bestimmen lässt. Als Beleg für den ersten Fall wird die englische Präposition in genannt, im zweiten Fall ist es die Präposition under (dt. unter), in der die topologischen, intrinsischen und vertikalitätsbezogenen Vorstellungen über die Lage eines Verweisobjekts verschmelzen (vgl. Levinson 2003: 72).

\section{Empirischer Teil der Arbeit}

\subsection{Datengrundlage und Ergebnisse in Bezug auf die wichtigsten Wortarten}

Um den genauen Anteil von Präpositionen festzulegen, muss man von dem gesamten Wortschatz die Anzahl der be-Verben bestimmen. Als Datengrundlage wurde das Wahrig Wörterbuch Digital 2007 herangezogen, zu dem die be-Verben aus Eroms (1980) noch hinzugefügt wurden. Insgesamt wurden 2334 Lexeme mit einem Präfix be-gefunden. Aus dieser Anzahl wurden 569 be-Verben (24\%) und 843 be-Nomina (35\%) aussortiert. Daher lässt sich feststellen, dass im heutigen Deutsch insbesondere der nominale Bereich mit be- präfigiert wird.

\subsection{Ergebnisse der Forschung nach dem Verhältnis der Simplexverben zu den präfigierten Verben}

Wir wenden uns nun unmittelbar den be-Verben zu und werfen einen Blick auf die Fähigkeit einzelner Wortklassen, das Präfix be- an sich zu binden. Die Ergebnisse werden in Tabelle 1 deutlich (s. unten).

Erstens darf es angenommen werden, dass den zusammengesetzten $b e$-Verben in der Mehrheit der Fälle eine Simplexform entsprechen wird. Diese Annahme ist insofern richtig, als es für die von den be-präfigierten Verben in

verwendet werden. „Ein Objekt kann maximal sechs verschiedene gerichtete Nachbarschaften haben“ (Wunderlich 1986: 12, zit. nach Vater 1996: 59).

${ }^{25}$ Die Begriffe Verweisobjekt und Bezugsobjekt werden in Vater in Anlehnung an Levelt (1986: 188, zit. nach Vater 1996: 51) gebraucht. In unserer Begriffsbestimmung sind sie mit den deutschen Vordergrund/Hintergrund gleichwertig. 
64\% der Fälle in der Tat eine Simplexform gibt. Dabei ist die Tatsache wichtig, dass manche von ihnen als veraltet (antragen, gatten) angesehen werden, in der Umgangssprache (zahlen, trillern) benutzt werden oder in einem figurativen Gebrauch der Bedeutung verwendet werden (pflanzen, rauschen, schleusen). Was die Nomina zusammen mit den Adjektiven betrifft, so können sie mittels Präfigierung die Komposita in 18\% aller Fälle bilden.

Tabelle 1. Verhältnis einzelner Wortklassen zu der Präfigierung mittels be-

\begin{tabular}{|l|l|l|}
\hline Nomen/Adjektiv als Basis & 11 & $2 \%$ \\
\hline Adjektiv als Basis & 26 & $5 \%$ \\
\hline lexikalisierte Paraphrasierungen von be-Verben & 32 & $6 \%$ \\
\hline Nomen als Basis & 64 & $11 \%$ \\
\hline Simplexform ohne Valenzpräposition & 103 & $18 \%$ \\
\hline Simplexform mit einer Valenzpräposition aufgrund unmöglicher Konversion & 143 & $25 \%$ \\
\hline Simplexform mit einer Valenzpräposition aufgrund möglicher Konversion & 176 & $31 \%$ \\
\hline be- Verben, die nicht berücksichtigt werden & 14 & $2 \%$ \\
\hline Insgesamt & 569 & $100 \%$ \\
\hline
\end{tabular}

\subsection{Ergebnisse der Forschung aus der Sichtweise der Raum- -Linguistik, bzw. grammars of space}

Tabelle 2 soll den Anteil von Präpositionen bestimmen, die für das Präfix $b e$-Räumlichkeit kennzeichnen. Einige der Verben lassen auch zwei Präpositionen zu, deswegen ist die gesamte Angabe höher.

Tabelle 2. Anteil von einzelnen Präpositionen der Simplexverben

\begin{tabular}{|l|c|c|}
\hline $\begin{array}{c}\text { Anzahl von den Verben, die eine Konversion in eine Simplexform mittels } \\
\text { einer Präposition zulassen / ihre Valenzpräpositionen }\end{array}$ & 177 & \\
\hline auf & 78 & $40 \%$ \\
\hline an & 47 & $24 \%$ \\
\hline über & 38 & $19 \%$ \\
\hline in & 17 & $9 \%$ \\
\hline nach & 9 & $4 \%$ \\
\hline wegen & 6 & $3 \%$ \\
\hline zu & 2 & $1 \%$ \\
\hline Insgesamt & 197 & $100 \%$ \\
\hline
\end{tabular}


Laut Vater (1996: 40) „[bezeichnen] im Deutschen [...] auf und über die konzeptuelle Relation ÜBER". Aus der Tabelle geht somit hervor, dass knapp zwei Drittel der be-Verben das Raumkonzept über bezeichnen. Wie im vorigen Kapitel gesagt wurde, stellt die aufrechte Achse eine Besonderheit dar, weil sie auf der Schnittstelle zwischen topologischer und intrinsischer Beschreibung mittels der Festlegung eines Referenzrahmens steht. Vater stellt weiter in Bezug auf die sogenannte kanonische Position von Gegenständen fest:

Die vertikale Dimension unterscheidet sich von den beiden andern insofern, als der Standpunkt des Sprechers bei der deiktischen Orientierung keine Rolle spielt. [...] Position des Sprechers und Orientierung seines Körpers sind also irrelevant. Wesentlich ist die wahrgenommene Vertikalität, so wie die wahrgenommene Horizontalität bei links und rechts (Vater 1996: 62).

Aufgrund der von Vater dargelegten Bilder lässt sich schließen, dass es von keiner Bedeutung ist, ob man steht, an der Seite liegt bzw. auf dem eigenen Kopf steht, die aufrechte Achse wird immer auf dieselbe Art und Weise betrachtet.

Die Präposition an, die noch einen bedeutenden Anteil an der Semantik des Präfixes be- aufweist, wird sowohl von Herweg (1989) als auch von Levinson (2003) als exemplarisches Beispiel für die topologische Positionierung bezeichnet. Um die Semantik von an besser zu verdeutlichen, ziehen wir folgende Belege in Betracht:

(16a) Peter arbeitet am Schreibtisch.

(16b) Peter arbeitet beim Schreibtisch.

„[Beleg 16a] wird im Allgemeinen so aufgefasst, dass zwischen Peter und dem Schreibtisch nicht nur eine lokale, sondern auch eine funktionale Beziehung besteht - nämlich dass der Schreibtisch mit Peters Arbeit zu tun hat - was bei b nicht der Fall ist" (Vater 1996: 67). Vater entwickelt die Räumlichkeit von an zu bei aufgrund Herwegs Überlegungen insoweit, als er das allgemeinere Konzept wie Kontakt (Berührung) ins Auge fasst: „[...] dass ,Kontakt zwar in der Semantik von bei ausgeschlossen ist, in der Semantik von an aber nicht notwendig vorhanden ist, sondern aufgrund konzeptueller und pragmatischer Faktoren zustande kommt" (Vater 1996: 72). Aus dem Gesagten bestätigt sich auch Hittmairs Annahme (vgl. 1882: 17), die besagt, dass be- den transitiven, hingegen bei- den intransitiven Verben einen Vorzug geben mag. Dabei kann gesagt werden, dass die Annäherung der Tätigkeit, die mittels Präfixes geschieht, den ,Kontakt' ausdrückt, der im Falle von bei- nicht zustande kommt. 


\section{Schlusswort}

Die hier angeführten Ergebnisse der statistischen Aufzählung von Valenzpräpositionen der Simplexverben lassen unseren Blick eher auf konzeptuell geprägte Ansätze richten. Die Räumlichkeit, die sich im Präfix be- wederspiegelt, liegt einer höheren konzeptuellen Organisation zugrunde. Die Räumlichkeit bildet eine Grundlage für die Metaphorisierung, die als ein Teilprozess der Grammatikalisierung angesehen werden kann. Da die Metaphorisierung im Bereich ,Raum-Zeit-Ursache' (Schulze 2010: 17) erfolgt, sollen die hier vorgestellten Überlegungen als Ausgangspunkt für weitere Erwägungen verstanden werden. Man kann hier annehmen, dass in einigen Partizipialbildungen von be-Verben, die man ausschließlich adjektivisch verwendet, die Metaphorisierung einen Schritt weitergebracht werden kann.

Eine gewisse Abstraktion der lokalen Grundbedeutung des Präfixes bewird bereits bei Hittmair (1882: 60) erwähnt. Die Fähigkeit des Präfixes benicht nur eine lokale, sondern auch eine lokal-abstrakte Gruppe (z.B. besiegen - siegen über) der Verben zu bilden, eröffnet weitere Fragestellungen. Diesbezüglich soll überlegt werden, welche Präpositionen eine höhere Abstraktion bzw. einen höheren Grad an Entkonkretisierung aufweisen können. Meines Erachtens wird dies besonders in der Gruppe IV (vgl. Eroms 1980), die sich als be-Verben mit einem fortschreitenden Grade der Grammatikalisierung beschreiben lässt, ersichtlich. Aus diesem Grunde wäre ein tieferer Blick in die ,Raumlinguistik' bzw. grammars of space, die einen übertragenen Gebrauch der Präpositionen anstreben, wünschenswert. Nicht zuletzt soll noch die Zahl von Satzpaaren, in denen man sogenannte, grammatische Relationen' berücksichtigt, in Betracht gezogen werden. Darüber hinaus werden auch die Listen von be-Verben sowohl aus dem Alt- als auch dem Neuhochdeutschen besonders hilfreich, um die Grammatikalisierung des Präfixes bemöglichst vollständig zu erfassen. Was die syntaktische Umstellung, die die $b e$-Verben veranlassen, angeht, soll man des Weiteren an dem Begriff Hintergrund-Diathese arbeiten.

\section{Literatur}

BRINKMANN, Ursula (1997): The 'Locative Alternation' in German: Its Structure and Acquisition. Amsterdam.

CROFT, William/CRUSE, D. Alan (2004): Cognitive Linguistics. Cambridge.

EROMS, Hans-Werner (1980): Be-Verb und die Präpositionalphrase: Ein Beitrag zur Grammatik der deutschen Verbalpräfixe. Heidelberg. 
GIVÓN, Talmy (1984): Syntax: A Functional-Typological Introduction. Amsterdam/ Philadelphia.

GÜNTHER, Hartmut (1971): Das System der Verben mit BE- in der deutschen Sprache der Gegenwart. Ein Beitrag zur Struktur des Lexikons der deutschen Grammatik. Tübingen.

HITTMAIR, Anton (1882): Die Partikel be in Mittel- und Neuhochdeutschen Verbalcomposition. Wien.

LEVINSON, Stephen C. (2003): Space in Language and Cognition. Cambridge.

LEVINSON, Stephen C./ WILKINS, David P. (2006): The Background to the Study of the Language of Space. In: Levinson, Stephen C./Wilkins, David P. (Hg.): Grammars of Space. Explorations in Cognitive Diversity. Cambridge, S. 1-22.

LINKE, Angelika/NUSSBAUMER, Markus/PORTMANN, Paul R. (2004): Studienbuch Linguistik. Tübingen.

MAYLOR, B. Roger. (2002): Lexical Template Morphology: Change of State and the Verbal Prefixes in German. Amsterdam.

TALMY, Leonard (2004): Toward a Cognitive Semantics 1. Cambridge.

TUHÁRSKA, Zuzana (2011): Die Analyse der semantisch kognitiven Ebene der Fachsprache. Hamburg.

VATER, Heinz (1996): Einführung in die Raum-Linguistik. 3. überarb. Auflage. Hürth.

WAHRIG WÖRTERBUCH (2007): Wahrig.digital.2.1. Gütersloh/München.

WILDGEN, Wolfgang (2008): Kognitive Grammatik. Klassische Paradigmen und neue Perspektiven. Berlin.

ZIFONUN, Gisela (1976): Rezension von: Hartmut Günther, Das System der Verben mit BE- in der deutschen Sprache der Gegenwart. Ein Beitrag zur Struktur des Lexikons der deutschen Grammatik. In: Zeitschrift für germanistische Linguistik 4.2/1976. Deutsche Sprache in Gegenwart und Geschichte, S. 248-253.

\section{Internetquellen}

http://schulzewolfgang.de/material/kasustypologie.pdf (01.08. 2014).

http://schulzewolfgang.de/material/Prasyn.pdf (2.03. 2014).

http://www.ats.uni-muenchen.de/downloads/schulze_down/wpct1.pdf (3.08.2014). http://wschulze.userweb.mwn.de/BB/morph_bb2.pdf (29.07.2014).

http://wschulze.userweb.mwn.de/WS0708/gksynt2.pdf (25.07.2014).

https://www.academia.edu/1802279/The_Grammaticalization_of_Antipassives

(1.08.2014). 